\title{
A Patient with Platelets Deficient in Glycoprotein VI That Lack Both Collagen-induced Aggregation and Adhesion
}

\author{
Masaaki Moroi, Stephanie M. Jung, Minoru Okuma, * and Koichi Shinmyozu* \\ Department of Biochemistry II, Jichi Medical School, Tochigi, Japan 329-04; *the First Department of Internal Medicine, \\ Faculty of Medicine, Kyoto University, Kyoto, Japan 606; and $\ddagger^{\ddagger}$ the Department of Internal Medicine, \\ Kagoshima City Hospital, Kagoshima, Japan 892
}

\begin{abstract}
Molecular level studies on platelets deficient in collagen-induced aggregation provide evidence for identifying possible platelet collagen receptors. We investigated platelets from a patient with mild bleeding time prolongation, but otherwise normal coagulation data. Her platelets lacked collagen-induced aggregation and adhesion, but retained normal aggregation and release by other agonists. Labeling her platelets with ${ }^{125}$ I or ${ }^{3} \mathrm{H}$ and analysis by SDS-PAGE/autoradiography showed normal levels of glycoproteins Ia, Ib, IIa, IIb, IIIa, and IV. However, there were significantly decreased incorporations of both radioactivities into a 61-kD membrane glycoprotein (GP), which was identified as GPVI from its mobility on unreduced-reduced, two-dimensional SDS-PAGE. Sugiyama et al. (1987. Blood. 69: 1712) reported that the serum from an idiopathic thrombocytopenic purpura (ITP) patient contained an antibody against a 62-kD platelet protein. Our patient's platelets lacked the antigen for the ITP patient's antibody, demonstrating that the ITP serum contains a specific antibody against GPVI. The patient's parents' platelets contained $\sim 50 \%$ the normal amount of GPVI, but still had normal collagen-induced aggregation and adhesion. The patient's platelets did not bind to types I and III collagen fibrils. Our results suggest that GPVI functions as a collagen receptor.
\end{abstract}

\section{Introduction}

Because the adhesion of platelets to subendothelial components is the first reaction of thrombus formation and one of the subendothelial components that reacts with platelets is collagen, the specific interaction of platelets with collagen is a crucial yet elusive topic of investigation for numerous platelet researchers. Many platelet proteins have been proposed to be components that interact with collagen on the cell surface. Collagen:glucosyl transferase (1), fibronectin (2), glycoprotein (GP) ${ }^{1}$ IIb (3), 65-kD protein (4), 61-kD protein (5), Factor XIII

The present address of Dr. Moroi and Dr. Jung is Department of Protein Biochemistry, Life Science Institute of Kurume University, Research Center Building, Kurume Research Park, 2432-3 Aikawacho, Kurume-shi, Fukuoka 830, Japan.

All correspondence should be addressed to Masaaki Moroi at the above address.

Received for publication 24 February 1989 and in revised form 8 June 1989.

1. Abbreviations used in this paper: GP, glycoprotein; ITP, idiopathic thrombocytopenic purpura; NEM, $N$-ethylmaleimide.

J. Clin. Invest.

(c) The American Society for Clinical Investigation, Inc.

0021-9738/89/11/1440/06 \$2.00

Volume 84, November 1989, 1440-1445
(6), and thrombospondin $(7,8)$ were proposed to be platelet receptors for collagen, but none were confirmed to be such. Recently GPIa was identified as a receptor for collagen from studies using GPIa-deficient platelets (9) and MAb against GPIa (10). In this context, a patient whose platelets have no collagen-induced aggregation and adhesion, and who shows a deficiency of a specific protein, would provide strong evidence that this protein is a collagen receptor. Previously we presented a case report on a patient whose platelets specifically lacked collagen-induced aggregation and were unable to adhere to collagen fibrils (11). In this report we analyzed the membrane GPs of this patient's platelets and showed that her platelets lacked a 61-kD GP, which we identified as GPVI of the platelet membrane. These results indicate that GPVI is involved in the interaction between platelets and collagen.

\section{Methods}

Case history. The patient was a 26-yr-old woman in 1985 when she first came to our hospital to undergo a blood examination because of purpura on her body. She had an ongoing tendency to develop purpura since she was a young child, occasionally had hypermennorrhea, and sometimes required several hours to stop a nosebleed. However, she never had any severe bleeding problems.

Coagulation studies showed that the patient had normal levels of all coagulation factors, a normal prothrombin time, and a normal activated partial thromboplastin time. The Factor VIII activity and the activity of $\mathrm{vWf}$ (measured by antigenic activity and ristocetin cofactor) were normal. Her bleeding time was slightly prolonged $(8.5 \mathrm{~min}$ as compared with 2-6 min for normal individuals). The clinical data for her platelet activity are summarized in Table I. The only platelet abnormality was inability to adhere to collagen fibrils and lack of collagen-induced aggregation. Aggregations by other agents and the release reaction were all normal.

The patient was not the child of a consanguineous marriage, and her parents showed normal values for the tests in which the patient exhibited abnormalities.

Platelet preparations. Whole blood was drawn from the antecubital vein of the patient, the parents of the patient, and healthy volunteers into 0.1 vol of $3.8 \%$ sodium citrate. The whole blood was centrifuged at $700 \mathrm{~g}$ for $5.5 \mathrm{~min}$, and the resultant platelet-rich plasma (PRP) was used for the following analyses.

For platelet GP analysis, the PRP was added to 0.1 vol of $0.5 \mathrm{M}$ EDTA solution, pH 7.4, and then sedimented by centrifugation at $1,000 \mathrm{~g}$ for $10 \mathrm{~min}$. The resultant pellet was washed twice with buffer A, which consisted of $6.85 \mathrm{mM}$ citrate, $130 \mathrm{mM} \mathrm{NaCl}, 4 \mathrm{mM} \mathrm{KCl}, 5.5$ $\mathrm{mM}$ glucose, and $5 \mathrm{mM}$ EDTA, $\mathrm{pH}$ adjusted to 6.5. The washed platelets were dissolved by adding a solution of $2 \%$ SDS, $8 \mathrm{M}$ urea, and $10 \mathrm{mM} N$-ethylmaleimide (NEM), followed by heating at $85^{\circ} \mathrm{C}$ for $5 \mathrm{~min}$.

Platelet aggregation and the release reaction were monitored by a lumiaggregometer (Chrono-Log Corp., Havertown, PA). $0.45 \mathrm{ml}$ PRP was mixed with $50 \mu \mathrm{l}$ luciferase-luciferin reagent (Chrono-Log Corp.) and the stimulants were added. For some assays the luciferase-luciferin reagent was omitted and only the aggregation reaction was monitored. 
Table I. Clinical Data of the Patient

\begin{tabular}{|c|c|c|c|c|}
\hline & Patient & Mother & Father & $\begin{array}{c}\text { Normal } \\
\text { individuals }\end{array}$ \\
\hline Platelet count $\left(\times 10^{-4} / \mu l\right)$ & 28.5 & 26.1 & 27.3 & $13-40$ \\
\hline Bleeding time (Ivy method, min) & 8.5 & 4.0 & 4.5 & $2-6$ \\
\hline Clot retraction $(\%)$ & 48 & ND & ND & $44-67$ \\
\hline Platelet adhesion to collagen (\%) & 1.3 & 37.7 & 31.1 & $23-42$ \\
\hline $\begin{array}{l}\text { Platelet release ( } \mathrm{nmol} \mathrm{ADP} / \\
\left.\quad 10^{9} \mathrm{cells}\right)\end{array}$ & 23.1 & ND & ND & $11-37$ \\
\hline \multicolumn{5}{|l|}{ Platelet aggregation (\%) } \\
\hline $\operatorname{ADP}(2 \mu M)$ & 81 & ND & ND & $30-60$ \\
\hline Collagen $(2 \mu g / m l)$ & 3 & 68 & 51 & $50-75$ \\
\hline Epinephrine $(1 \mu \mathrm{g} / \mathrm{ml})$ & 83 & ND & ND & $55-90$ \\
\hline AA $(100 \mu g / m l)$ & 100 & ND & ND & $50-85$ \\
\hline Ristocetin $(1.2 \mathrm{mg} / \mathrm{ml})$ & 81 & ND & ND & $60-90$ \\
\hline Bovine fibrinogen $(1.5 \mathrm{mg} / \mathrm{ml})$ & 94 & ND & ND & $60-90$ \\
\hline Thrombin $(0.2 \mathrm{U} / \mathrm{ml})$ & 95 & ND & ND & $70-90$ \\
\hline
\end{tabular}

Data taken from K. Shinmyozu et al. (11).

The following agonists were used: collagen (Hormon-Chemie, Munich, FRG); human type I and type III collagens (Fuji Chem. Ind., Ltd., Toyama, Japan); bovine types I, III, IV, and V collagens (Koken, Ltd., Tokyo, Japan); ristocetin (H. Lundbeck \& Co., Copenhagen, Denmark); and ADP (Sigma Chemical Co., St. Louis, MO).

Platelets were radiolabeled according to the method of Phillips (12) using lactoperoxidase (Sigma Chemical Co.). After washing twice with buffer $A$ the platelets were dissolved in SDS/urea/NEM solution as described above. Platelets were also tritium-labeled by the periodate method of Berndt and Phillips (13). After washing with $1 \mathrm{mM}$ EDTA, $0.15 \mathrm{M} \mathrm{NaCl}$, and $10 \mathrm{mM}$ Hepes, $\mathrm{pH} 7.6$, the labeled platelets were dissolved in SDS/urea/NEM solution. The solubilized platelets were analyzed by two-dimensional gel electrophoresis (described in the next section), and the gel was dried in the presence or absence of $\mathrm{EN}^{3} \mathrm{HANCE}$ (New England Nuclear, Boston, MA) for the detection of
${ }^{3} \mathrm{H}$ or ${ }^{125} \mathrm{I}$, respectively, and exposed to Fuji x-ray film (Fuji Photo Film Co., Ltd., Kanagawa, Japan) for $\sim 1$ wk at $-70^{\circ} \mathrm{C}$.

Gel electrophoresis and related techniques. The Laemmli method (14) was used for SDS-PAGE. Two-dimensional, unreduced-reduced gel electrophoresis was performed as described previously $(15,16)$.

The electrophoresed gels were also electrophoretically transferred to nitrocellulose membranes (BA85; Schleicher \& Schuell, Inc., Keene, $\mathrm{NH})(17)$ and stained with antibody. The transferred and washed nitrocellulose strips were reacted with serum from the idiopathic thrombocytopenic purpura (ITP) patient described by Sugiyama et al. (18), which was diluted 100 -fold with $10 \mathrm{mM}$ Tris, $0.14 \mathrm{M} \mathrm{NaCl}$, and $0.5 \%$ BSA, pH 7.4, washed, incubated with peroxidase-conjugated antihuman IgG antibody $(0.5 \mu \mathrm{g} / \mathrm{ml}$; E. Y. Laboratories, Inc., San Mateo, $\mathrm{CA}$ ), and finally reacted with $0.02 \% 3,3^{\prime}$-diamino-benzidine and $0.005 \% \mathrm{H}_{2} \mathrm{O}_{2}$ to visualize the proteins.

The amounts of GPVI were measured by densitometry of the antibody-stained nitrocellulose paper (19). On the same gel, different amounts of whole platelet proteins from a normal individual (chosen as the standard, reference sample) and 2- $\mu \mathrm{g}$ samples of whole platelet proteins from the patient, her parents, and other controls (normal volunteers) were analyzed by SDS-PAGE/immunostaining with ITP serum as described above; the amounts of GPVI were determined by densitometry, measuring the GPVI peak in terms of arbitrary units of area. A standard curve was made by plotting the areas obtained for different amounts of the reference sample against the amount of applied reference sample. The relative amounts of GPVI in the samples from the patient, her parents, and controls were calculated from their respective GPVI peak areas and the standard curve and expressed as a percentage of the reference sample.

Adhesion of platelets to collagens. Adhesion of platelets to collagen was measured by the method described by Mant (20). PRP was separated as described above and mixed with autologous platelet-poor plasma so that the platelet count was $2.5 \times 10^{8} / \mathrm{ml}$, and then a sufficient amount of $0.5 \mathrm{M}$ EDTA was added to bring the final EDTA concentration to $5 \mathrm{mM}$. $0.5 \mathrm{ml}$ of this PRP was stirred at $37^{\circ} \mathrm{C}$ in an aggregometer, mixed with 16.7 or $33.3 \mu$ l of collagen solution ( 3 $\mathrm{mg} / \mathrm{ml}$ ), and incubated for $10 \mathrm{~min}$ with stirring. The platelet count was measured by a Coulter counter (model ZbI; Coulter Electronics, Inc., Hialeah, FL), and the adherent platelets were calculated by subtracting the number of platelets in PRP treated with collagen from that in PRP incubated without collagen.
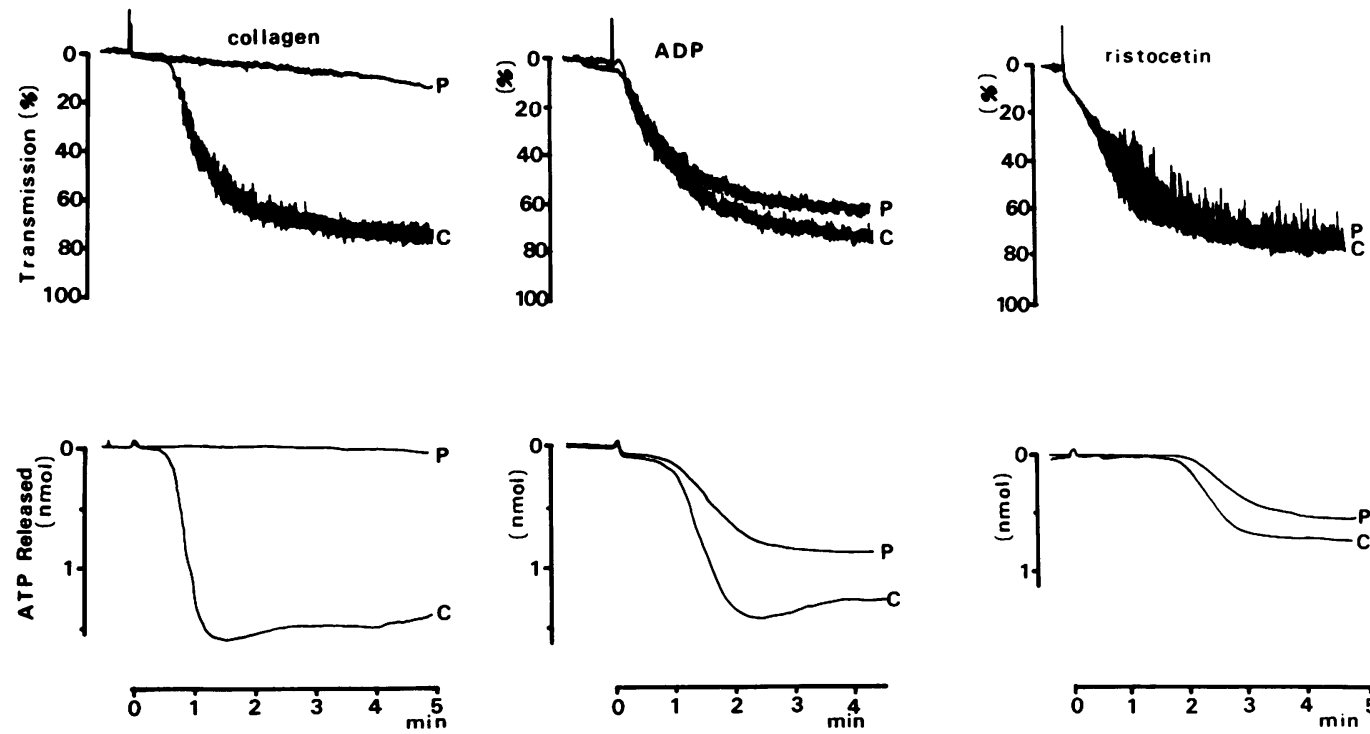

Figure 1. Aggregation and release reaction of platelets from the patient and a normal individual. PRP from the patient $(P)$ and a normal individual (C) were stimulated with collagen $(5 \mu \mathrm{g} / \mathrm{ml})$, ADP $(10 \mu \mathrm{M})$, or ristocetin $(1.5$ $\mathrm{mg} / \mathrm{ml}$ ) and monitored by the lumiaggregometer. The upper traces show the aggregation profiles and the lower panels show the respective curve for the luminescence due to the platelet-released ATP. The amount of ATP release was estimated by adding a known amount of ATP at the end of the monitoring period and measuring the luminescence change. 


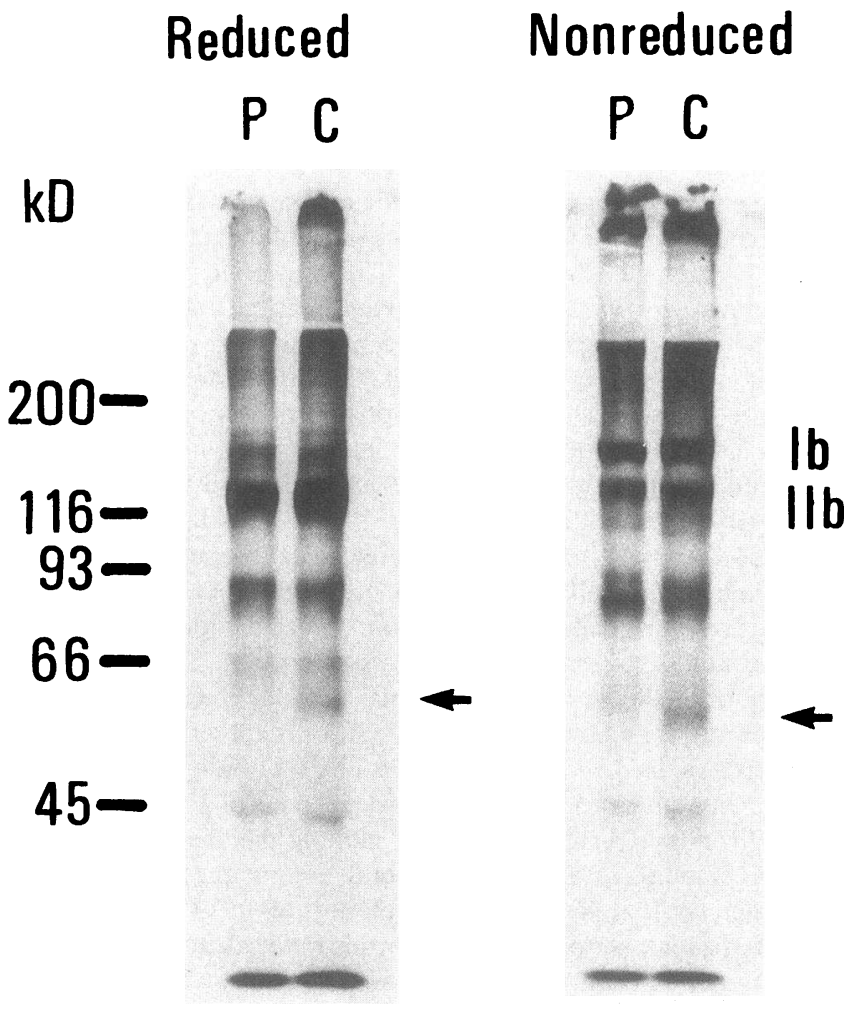

Figure 2. Fluorograms of SDS-PAGE gels of platelets that were surface-labeled with ${ }^{3} \mathrm{H}$. Solubilized ${ }^{3} \mathrm{H}$-labeled platelets from the patient $(P)$ and a normal individual $(C)$ were electrophoresed on $7.5 \%$ Laemmli gels under reduced or nonreduced conditions. A faint band (arrow) present in the normal platelets was decreased in the patient's platelets.

\section{Results}

Platelet aggregation. As indicated in Table I, the patient's platelets had normal aggregation responses to ADP, epinephrine, AA, ristocetin, bovine fibrinogen, and thrombin, but showed a profound decrease in collagen-induced aggregation. Some of the aggregation profiles shown in Fig. 1 also demonstrate that the patient's platelets can release ATP normally.
The normal release of ATP by the patient's platelets was further confirmed by measuring released ADP by the method of Weiss et al. (21; Table I). We also determined if the patient's platelets could aggregate in response to various kinds of purified collagens (human types I and III and bovine types I, III, IV, and V), and found that her platelets did not aggregate in the presence of any of these collagens at concentrations that aggregated normal platelets (data not shown).

Analyses of GPS in the patient's platelets. The platelets from the patient and a normal subject were tritium-labeled and analyzed by SDS-PAGE. The fluorograms in Fig. 2 indicate that a faint band with a molecular mass of $\sim 61 \mathrm{kD}$ is missing from the patient's platelets.

The patient's platelets were also labeled with ${ }^{125} \mathrm{I}$ and analyzed by unreduced-reduced, two-dimensional SDS-PAGE (Fig. 3). The amounts of GPIa, GPIb, GPIIa, GPIIb, GPIIIa, and GPIV in the patient's platelets were similar to those of normal platelets. However, a spot with a molecular mass of $\sim 60 \mathrm{kD}$ (as indicated by the star in Fig. 3) is nonexistent or extraordinarily faint in the patient's platelets. The molecular mass of this GP, which was designated as GPVI according to the definition by Phillips (22), was calculated to be $61 \mathrm{kD}$ from one-dimensional SDS-PAGE (data not shown).

Identification of the deficient protein by antibody staining. Sugiyama et al. (18) reported a patient with ITP whose platelets lacked collagen-induced aggregation because her serum contained an antibody against a $62-\mathrm{kD}$ platelet protein. We examined the possibility that GPVI is the same protein as the $62-\mathrm{kD}$ protein, which is the antigen of the anti-platelet antibody of the ITP patient. The platelets from our GPVI-deficient patient, her parents, and normal individuals were analyzed by SDS-PAGE, transferred to nitrocellulose, and reacted with the diluted serum from the ITP patient. As indicated in Fig. 4, a main band with a molecular mass of $61 \mathrm{kD}$ was stained in the normal platelets. However, the patient's platelets lacked this band, which indicated that the antibody of the ITP patient was an antibody against GPVI, and the platelets of her parents showed a decrease in the contents of GPVI. Staining also detected a $260-\mathrm{kD}$ protein; this band can be ascribed to the $\mathrm{Fc}$ receptor of the platelet membrane (23) because normal human plasma also stained the same band, and the band was not stained when the sample was reduced (data not shown). How-

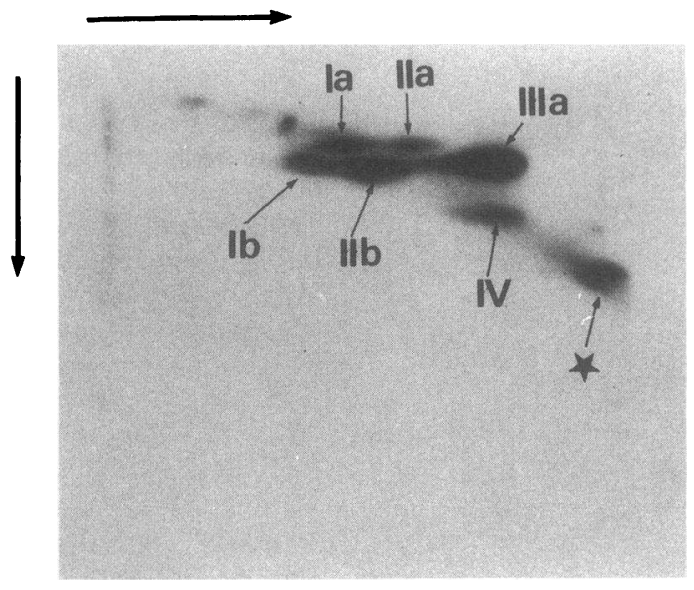

NOR MAL

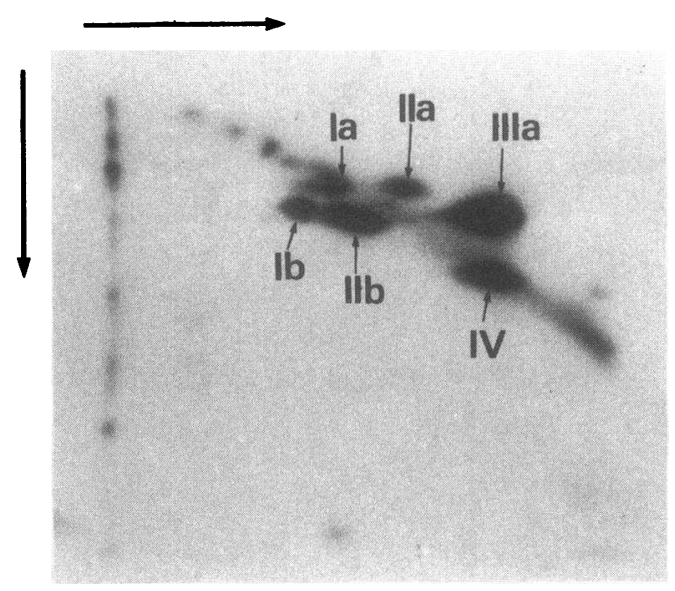

PATIENT
Figure 3. Autoradiograms of two-dimensional SDSPAGE gels of ${ }^{125}$ I-labeled platelets. First dimension, Nonreduced SDS-PAGE, $5.5 \%$ acrylamide; second dimension, reduced SDSPAGE, $7.5 \%$ acrylamide. The patient's platelets lacked the spot indicated by the star in the autoradiogram of the normal platelets. 

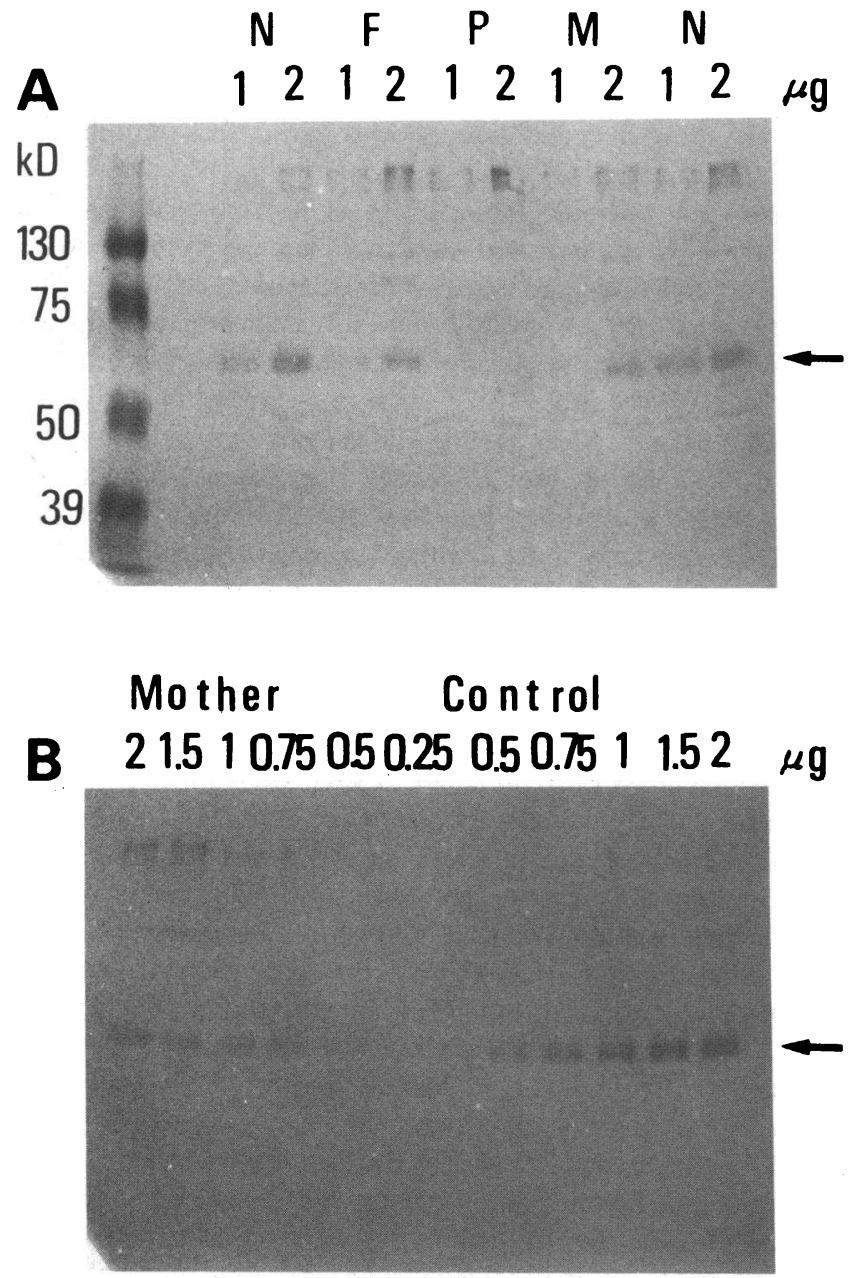

Figure 4. Immunoblotting analysis of GPVI. $A, 1$ or $2 \mu \mathrm{g}$ of whole platelet proteins from the patient $(P)$, the patient's mother $(M)$ and father $(F)$, and a normal individual were analyzed by SDS-PAGE, transferred to nitrocellulose sheets, and then reacted with serum from the ITP patient. GPVI is indicated by an arrow. Molecular weight marker proteins and their molecular weights are indicated on the left side. B, Quantitation of GPVI. Different amounts of whole platelet proteins from the patient's mother and the normal individual (control) were analyzed by SDS-PAGE, blot-transferred, and stained with serum from the ITP patient. Densities of the GPVI bands were measured and the relative content of GPVI in the platelets from the patient's mother was calculated by comparing its densities with those of the control platelets. The contents of GPVI in the mother's platelets was calculated to be $56 \%$ that of the normal control.

ever, we can not identify the faint bands with molecular masses of 130, 56, and $50 \mathrm{kD}$ in Fig. $4 \mathrm{~A}$. These bands may be stained because the plasma from the ITP patient that we used for the staining might contain small amounts of antibodies against some other platelet proteins. The amounts of GPVI were semi-quantitated with densitometry (Fig. $4 B$ ). The GPVI contents in the platelets from the patient's father and mother were calculated to be 51.4 and $56 \%$ in comparison to the normal, reference individual, respectively. The contents of GPVI in the other normal volunteers $(n=5)$ were $96.0 \pm 12.2 \%$ relative to the same reference individual.

The identification of the $62-\mathrm{kD}$ protein reported by Sugiyama et al. (18) as GPVI was also confirmed using the ability of the antibody from the ITP patient to induce aggregation. As indicated in Fig. 5, $2 \mu \mathrm{l}$ of plasma from the ITP patient could aggregate normal platelets, but this induced no reaction in the platelets from our patient. Control plasma that contained no antibody to the $62-\mathrm{kD}$ protein did not aggregate normal platelets. These experiments suggested that our patient lacked the reactive antigen which can be recognized by the antibody from the ITP patient.

Adhesion of the patient's platelets to collagen fibrils. The ability of the patient's platelets to adhere to collagen fibrils was examined using several types of purified collagens. Table II shows that normal platelets adhere to bovine type III and human types I and III, but the patient's platelets fail to adhere to any of them. Other types of collagen, bovine types I, IV, and $\mathrm{V}$, did not show significant binding to either the normal platelets or those from our patient. This may be because these collagen preparations must be present at higher concentrations to bind a significant number of platelets in our assay system, since substantially higher concentrations of these collagens were required to induce platelet aggregation relative to the required amounts of bovine type III and human types I and III collagens.

Table I shows the adhesion data that were obtained by the same method but with the use of horse tendon collagen, which is mainly composed of type I collagen. Again, the patient's platelets showed a lack of adhesion to these collagen fibrils and the platelets from her parents adhered normally.
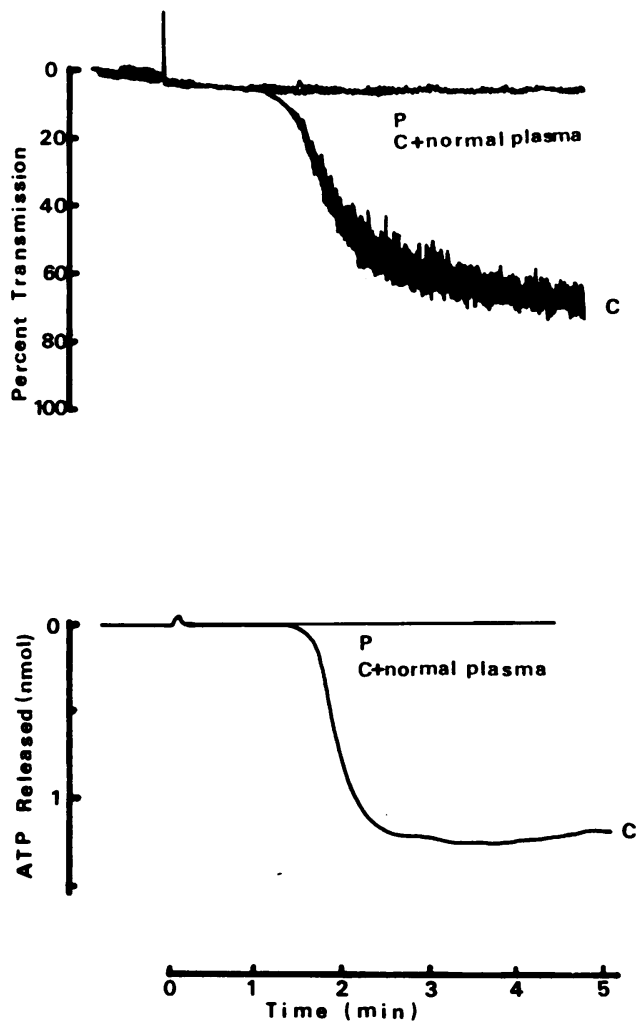

Figure 5. Platelet aggregation and release induced by plasma from the ITP patient. Plasma from the ITP patient was added to normal $(C)$ and patient's $(P)$ PRP, and the aggregation and release reaction were monitored. Plasma from the normal individual was also added to the normal PRP (control plasma). 
Table II. Platelet Adhesion to Collagen Fibrils

\begin{tabular}{lcr}
\hline & \multicolumn{2}{c}{ Adhered platelets } \\
\cline { 2 - 3 } Type of collagen & $\begin{array}{c}\text { Normal platelets } \\
(n=5)\end{array}$ & $\begin{array}{c}\text { Patient's } \\
\text { platelets }\end{array}$ \\
\hline & & $\%$ \\
Bovine type I & $6.5 \pm 8.3$ & -1.9 \\
Bovine type III & $25.1 \pm 11.8$ & 2.7 \\
Bovine type IV & $-0.3 \pm 5.8$ & -0.8 \\
Bovine type V & $0.3 \pm 3.3$ & 0.4 \\
Human type I & $30.6 \pm 16.5$ & 6.5 \\
Human type III & $68.4 \pm 12.4$ & 8.1 \\
\hline
\end{tabular}

Platelets that adhered to collagen fibrils were expressed as a percent of the original platelet count.

\section{Discussion}

Nieuwenhuis et al. first reported a patient whose platelets did not show aggregation induced by collagen and adhesion to collagen fibrils (9). The patient's platelets were found to have only $15-25 \%$ of the normal level of GPIa, which suggested that GPIa participated in the interaction with collagen on the platelet surface. Kunicki et al. (10) obtained evidence to support this hypothesis by using a MAb against GPIa and also demonstrated that GPIa is present as a complex with GPIIa. Handa et al. (24) reported a similar patient who lacked collagen-induced platelet aggregation and showed that the patient's platelets do not contain the GPIa/GPIIa complex. These results indicate that GPIa, as part of a complex with GPIIa, participates in the platelet adhesion to collagen fibrils and the resultant platelet aggregation.

However, there is still a possibility that another membrane protein would affect the interaction with collagen, since many proteins were suggested to be receptors of collagen. In the last few years, coagulation Factor XIII (6), thrombospondin (8), and GPIV (25) were suggested to function as collagen receptors. Since collagen fibrils interact with numerous proteins, it is difficult to identify a protein that actually works on the platelet surface. One of the most reliable ways to do this is to find a patient whose platelets specifically lack the interaction with collagen. Our patient was such an individual. Her platelets contained a normal level of GPIa, so the deficiency of another protein was suggested.

The aggregation studies (Fig. 1 and Table I) indicated that the platelets from our patient specifically lacked reactivity towards collagen. The patient's platelets aggregate normally in response to $\mathrm{ADP}$ and $\mathrm{AA}$, indicating that their aggregability and release reaction were normal. Although the ATP release in Fig. 1 shows that the patient's platelets released less ATP than the platelets from the particular normal individual used in this study, the data from the quantitative investigation given in Table I show that the release reaction of the patient's platelets were well within the normal range of levels (11). The patient's platelets did not show any aggregation in response to collagen at concentrations up to $10 \mu \mathrm{g} / \mathrm{ml}$. However, they apparently reacted with collagen, because a slight increase in transmission was observed. This transmission increase was dose dependent, ranging from 3 to $12 \%$ of light transmission (11). Part of this transmission increase could be ascribed to the polymerization of collagen. Platelets from the ITP patient with an autoantibody against GPVI (18) also showed a slight reactivity towards collagen; this was different from the behavior of the platelets from the GPIa-deficient patient who had defective collageninduced aggregation (9).

From the radiolabeling studies of normal platelets and those from the patient, the patient's platelets were found to have a very profound decrease or absence of a $61-\mathrm{kD}$ GP. The patient's platelets also showed markedly decreased binding towards collagen fibrils. Several other groups have suggested a GP with a similar molecular weight to be a receptor for collagen. Chiang and Kang purified a protein with a molecular mass of $65 \mathrm{kD}$ from human platelet membranes using an affinity column of immobilized chicken alpha 1 (I) collagen (4). Kotite and Cunningham identified a platelet membrane GP with a molecular mass of $61 \mathrm{kD}$ that bound specifically to insoluble collagen fibrils (5). Furthermore, Sugiyama et al. (18) reported a patient with ITP whose platelets had a defect in collagen-induced aggregation. The patient's serum contained an antibody that recognized a platelet membrane protein with a molecular mass of $62 \mathrm{kD}(18)$. A very interesting finding of the present study was the identification of GPVI, which was absent in our patient's platelets, as one of the candidates for these collagen receptors. Immunoblotting analysis using serum from the ITP patient described by Sugiyama et al. (18) as the first antibody showed that the patient's platelets also lacked the antigen that the ITP patient's antibody recognizes (Fig. 4). The identity of GPVI and the antigen of the ITP patient's antibody was also indicated from our aggregation studies (Fig. 5). These data indicated that the antibody from the ITP patient was an antibody against GPVI. Our data combined with the data on the ITP patient (18) strongly suggested that GPVI takes part in the interaction of platelets with collagen, and it may be one of the collagen receptors on the platelet membrane. The other proteins reported to be collagen receptors $(4,5)$ would be the same protein as GPVI since these proteins have similar characteristics as GPVI.

There have been no reports on the further characterization of GPVI since the designation of GPVI by Phillips and Agin (15) in their electrophoresis study and the report by Clemetson et al. (26) who observed two spots of GPVI with a small difference of molecular weight using two-dimensional gel electrophoresis and ${ }^{3} \mathrm{H}$-labeling techniques when they were analyzing GPs of platelets from Bernard-Soulier patients. We could not find such a GPVI doublet in our nonreduced SDS-PAGE system. Since our antibody did not react with reduced GPVI, we could not confirm Clemetson's observation.

Semi-quantitative analysis of the GPVI contents in the platelets from the patient's parents showed that both her parents had about half the amount of GPVI in comparison with normal platelets, which indicates that the patient's GPVI defect is inherited from both parents. However, the parents' platelets showed normal aggregability against collagen and normal adhesion to collagen fibrils. This would be because $50 \%$ GPVI is sufficient for platelets to interact with collagen fibrils.

When the aggregation profiles of the GPIa-deficient patient described by Nieuwehuis et al. (9) were compared with those of our patient, the former patient showed absolutely no reactivity to collagen, but our patient showed a very small extent of platelet aggregation under the usual conditions used for the aggregation test. Also, the GPIa-deficient individual had a 
more severe bleeding tendency, having a longer prolongation of bleeding time in comparison with our GPVI-deficient patient who showed only a mild bleeding tendency. These results suggest that GPIa would play a more significant role on the platelet surface during hemostasis than GPVI. However, the mechanism of the interaction between platelets and collagen that is a component of the subendothelium is still not fully understood because collagen has a macromolecular structure and would therefore be expected to interact with many proteins. The GPVI-deficient platelets described in this study would be a useful means to study the interaction between platelets and collagen.

\section{Acknowledgments}

This study was supported in part by Grants-in-Aid for Scientific Research on Priority Areas from the Ministry of Education of the Government of Japan, The Mochida Memorial Foundation for Medical and Pharmaceutical Research, and The Ichiro Kanehara Foundation.

\section{References}

1. Jamieson, G. A., C. L. Urban, and A. J. Barber. 1971. Enzymatic basis for platelet:collagen adhesion as the primary step in haemostasis. Nature New Biol. 234:5-7.

2. Bensusan, H. B., T. L. Koh, K. G. Henry, B. A. Murray, and L. A. Culp. 1978. Evidence that fibronectin is the collagen receptor on platelet membranes. Proc. Natl. Acad. Sci. USA. 75:5864-5868.

3. Tsunehisa, S., T. Tsuji, H. Tohyama, and T. Osawa. 1984. Interaction of human platelet membrane glycoproteins with collagen and lectins. Biochim. Biophys. Acta. 797:10-19.

4. Chiang, T. M., and A. H. Kang. 1982. Isolation and purification of collagen $\alpha 1$ (I) receptor from human platelet membrane. J. Biol. Chem. 257:7581-7586.

5. Kotite, N. J., and L. W. Cunningham. 1986. Specific adsorption of a platelet membrane glycoprotein by human insoluble collagen. $J$. Biol. Chem. 261:8342-8347.

6. Saito, Y., T. Imada, J. Takagi, T. Kikuchi, and Y. Inada. 1986. Platelet factor XIII: the collagen receptor? J. Biol. Chem. 261:13551358.

7. Lahav, J., M. A. Schwartz, and R. I. Hynes. 1982. Analysis of platelet adhesion with a radioactive chemical crosslinking reagent: interaction of thrombospondin with fibronectin and collagen. Cell. 31:253-262.

8. Kehrel, B., L. Balleisen, R. Kokott, R. Mesters, W. Stenziger, K. J. Clemetson, and J. van de Loo. 1988. Deficiency of intact thrombospondin and membrane glycoprotein Ia in platelets with defective collagen-induced aggregation and spontaneous loss of disorder. Blood. 71:1074-1078.

9. Nieuwenhuis, H. K., J. W. N. Akkerman, W. P. M. Houdijk, and J. J. Sixma. 1985. Human blood platelets showing no response to collagen fail to express surface glycoprotein Ia. Nature (Lond.). 318:470-472.

10. Kunicki, T. J., D. J. Nugent, S. J. Staats, R. P. Orchekowski, E. A. Wayner, and W. G. Carter. 1988. The human fibroblast class II extracellular matrix receptor mediates platelet adhesion to collagen and is identical to the platelet glycoprotein Ia-Ila complex. J. Biol. Chem. 263:4516-4519.

11. Shinmyozu, K., Y. Maruyama, M. Osame, and A. Igata. 1986. Collagen adhesion abnormality. Rinsho Ketsueki. 27:257-262.

12. Phillips, D. R. 1972. Effect of trypsin on the exposed polypeptides and glycoproteins in the human platelet membrane. Biochemistry. 11:4582-4588.

13. Berndt, M. C., and D. R. Phillips. 1981. Purification and preliminary physicochemical characterization of human platelet membrane glycoprotein V. J. Biol. Chem. 256:59-65.

14. Laemmli, U. K. 1970. Cleavage of structural proteins during the assembly of the head of bacteriophage T4. Nature (Lond.). 227:680-685.

15. Phillips, D. R., and P. P. Agin. 1977. Platelet plasma membrane glycoproteins: evidence for the presence of nonequivalent disulfide bonds using nonreduced-reduced two-dimensional gel electrophoresis. J. Biol. Chem. 252:2121-2126.

16. Moroi, M., and S. M. Jung. 1984. Selective staining of human platelet glycoproteins using nitrocellulose transfer of electrophoresed proteins and peroxidase-conjugated lectins. Biochim. Biophys. Acta. 798:295-301.

17. Towbin, H., T. Staehelin, and J. Gordon. 1979. Electrophoretic transfer of proteins from polyacrylamide gels to nitrocellulose sheets: procedure and some applications. Proc. Natl. Acad. Sci. USA. 76:4350-4354.

18. Sugiyama, T., M. Okuma, F. Ushikubi, S. Sensaki, K. Kanaji, and H. Uchino. 1987. A novel platelet aggregating factor found in a patient with defective collagen-induced platelet aggregation and autoimmune thrombocytopenia. Blood. 69:1712-1720.

19. Jung, S. M., N. Yoshida, N. Aoki, K. Tanoue, H. Yamazaki, and M. Moroi. 1988. Thrombasthenia with an abnormal platelet membrane glycoprotein IIb of different molecular weight. Blood. 71:915-922.

20. Mant, M. J. 1977. Platelet adherence to collagen: a simple, reproducible, quantitative method for its measurement. Thromb. Res. 11:729-737.

21. Weiss, H. J., P. A. Chervenick, R. Zalusky, and A. Factor. 1969. A familial defect in platelet function associated with impaired release of adenosine diphosphate. N. Engl. J. Med. 281:1264-1270.

22. Phillips, D. R. 1979. Surface labeling as a tool to determine structure-function relationships of platelet plasma membrane glycoproteins. Thromb. Haemostasis. 42:1638-1651.

23. Cheng, C. M., and J. Hawiger. 1979. Affinity isolation and characterization of immunoglobulin $\mathrm{G} \mathrm{Fc}$ fragment-binding glycoprotein from human blood platelets. J. Biol. Chem. 254:2165-2167.

24. Handa, M., H. Nagai, K. Kamata, H. Ando, Y. Araki, M. Yamamoto, Y. Kawai, Y. Ikeda, and K. Watanabe. 1988. Defect of GP Ia-Ila heterodimer complex in a patient whose platelets showed no response to collagen. Blood. 72:323a. (Abstr.)

25. Tandon, N. N., and G. A. Jamieson. 1987. Role of platelet membrane glycoprotein IV in platelet-collagen interaction: a microtiter assay to study platelet adherence. Thromb. Haemostasis. 58:303a. (Abstr.)

26. Clemetson, K. J., J. L. McGregor, E. James, M. Dechavanne, and E. F. Lusher. 1982. Characterization of the platelet membrane glycoprotein abnormalities in Bernard-Soulier syndrome and comparison with normal by surface-labeling techniques and high-resolution two-dimensional gel electrophoresis. J. Clin. Invest. 70:304-311. 